This item was submitted to Loughborough's Research Repository by the author.

Items in Figshare are protected by copyright, with all rights reserved, unless otherwise indicated.

\title{
Direct exterior orientation determination for a low-cost heritage recording system
}

PLEASE CITE THE PUBLISHED VERSION

http://dx.doi.org/10.1111/j.1477-9730.2012.00701.x

\section{PUBLISHER}

Wiley-Blackwell @ 2012 The Authors. The Photogrammetric Record @ 2012 The Remote Sensing and Photogrammetry Society and Blackwell Publishing Ltd.

\section{VERSION}

AM (Accepted Manuscript)

\section{LICENCE}

CC BY-NC-ND 4.0

\section{REPOSITORY RECORD}

Kirchhoefer, Melanie K., Jim H. Chandler, Rene Wackrow, and Paul Bryan. 2019. "Direct Exterior Orientation Determination for a Low-cost Heritage Recording System". figshare. https://hdl.handle.net/2134/14606. 
This item was submitted to Loughborough's Institutional Repository (https://dspace.lboro.ac.uk/) by the author and is made available under the following Creative Commons Licence conditions.

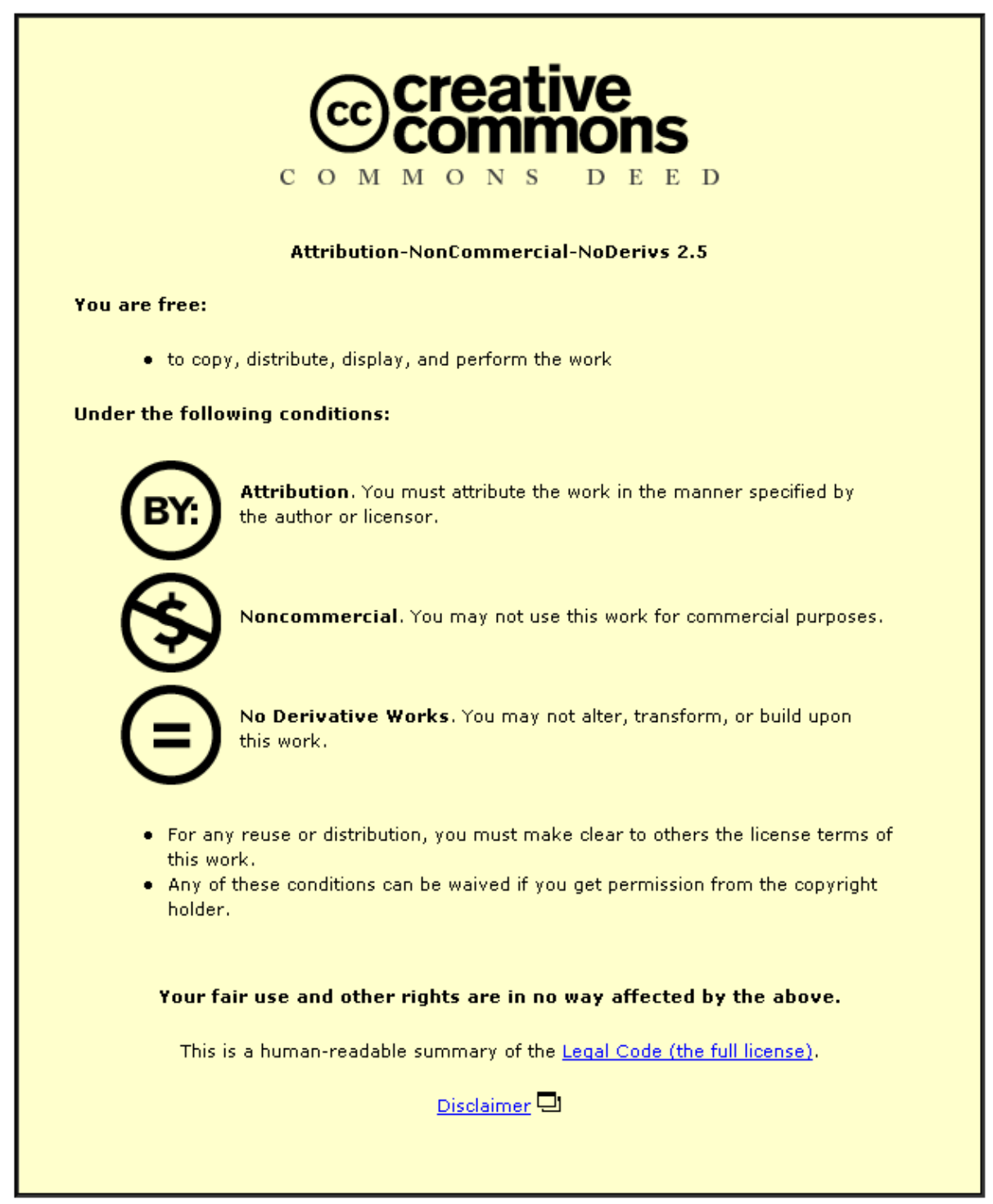

For the full text of this licence, please go to: http://creativecommons.org/licenses/by-nc-nd/2.5/ 


\title{
DIRECT EXTERIOR ORIENTATION DETERMINATION FOR A LOW COST HERITAGE RECORDING SYSTEM
}

\author{
MELANIE K. KIRCHHÖFER (m.k.kirchhoefer@lboro.ac.uk) \\ JIM. H. CHANDLER (j.h.chandler@lboro.ac.uk) \\ RENE WACKROW (r.wackrow@lboro.ac.uk) \\ Loughborough University, UK \\ PAUL BRYAN (paul.bryan@english-heritage.org.uk) \\ English Heritage, York, UK
}

\begin{abstract}
Close range photogrammetry has proved effective as a cost effective and easy to use method for cultural heritage recording. Off-the-shelf digital cameras can be used to rapidly acquire data at low cost, allowing non-experts to become more involved in their local heritage, an important aspiration identified in this collaboration between English Heritage and Loughborough University. To achieve this while also allowing for measurements in a 3D national reference system, a recording system has been developed that is capable of deriving the exterior orientation during exposure directly and cheaply. The system comprises a digital camera, a small 3D orientation sensor and a GNSS antenna, assembled in a frame that allows calibration of the offsets between the components. Test field data was used to investigate calibration precision and stability as well as achievable absolute and relative accuracy. The results of data analysis indicate that the system can meet the accuracy requirements for medium accuracy heritage recording, even with slightly unstable offset calibration values.
\end{abstract}

KEYWORDS: close range photogrammetry, cultural heritage recording, orientation sensor, MEMS, low-cost, GNSS receiver

\section{INTRODUCTION}

CULTURAL HERITAGE is at a constant risk by neglect and decay, deliberate destruction and damage due to social and economic progress, disasters, and armed conflict (UNESCO, 1972; Power of Place Office and English Heritage, 2000). From this risk, an increased need to record spatial information can be recognised. Comprehensive and accurate 
documentation can attenuate the risk of heritage loss and, in the worst case, serve as a basis for reconstruction (Palumbo and Ogleby, 2004).

Close range photogrammetry has proven to be well suited for heritage recording in the past (Clowes, 2002; Arias et al., 2005; 2005; Yilmaz et al. 2007). Indeed, the International Committee for Documentation of Cultural Heritage (CIPA) was established for exploring this potential (CIPA, 2010). Recent advances in digital technology, cheaper equipment and faster means of data processing have accelerated this trend. With proper calibration consumer-grade digital cameras can achieve accuracy levels that are sufficient for many heritage recording tasks (Bosch et al., 2005; Wackrow et al., 2007). Whilst recognising the desirability to record data within a 3D national reference system, establishing known coordinated target points for exterior orientation estimation remains time consuming and costly and requires surveying expertise. Particularly in times of cost cutting, high cost can prevent cultural heritage from being adequately recorded. Direct exterior orientation estimation for close-range applications could overcome the problem by avoiding expensive target point surveys and enabling non-experts to record cultural heritage within an appropriate national reference system. By this means the cost is reduced even further by the possibility of employing volunteers (Bryan and Chandler, 2008).

Devices that can be used for direct exterior orientation determination in close range photogrammetry include the small-size and low-cost orientation and position sensors that have emerged on the marked in recent years. Orientation sensors based on Micro Electro Mechanical Systems (MEMS) technology have already been utilised in connection with mobile mapping projects and photogrammetry (Niu et al, 2006; Guarnieri et al., 2008). Although their accuracy is lower than that of their large-size counterparts, the results look promising. Schwieger and Gläser (2005) and Wing et al. (2005) have shown that positioning with current low-cost, handheld GNSS devices does not meet the requirements for some applications of close range photogrammetry. However, it was also demonstrated that there is potential for improvements in the future. One example is the announcement by GENEQ Inc. to release a small-size, high-accuracy GNSS receiver (SXBlue III) that is available for a much lower cost (approximately £3,150) than conventional survey-grade GNSS receivers (GENEQ Inc., 2010).

This paper presents the development and testing of a low-cost recording system for cultural heritage recording that utilises a low-cost orientation sensor and GNSS for direct exterior orientation determination. After a review of work related to low-cost heritage recording, the recording system and its components are presented. This is followed by a description of the offset calibration procedure and performance testing, addressing calibration reliability and stability, as well as achievable accuracy. After discussing the results of recording system tests, the concluding section indicates the potential of the method.

\section{RELATED WORK}

In Bosch et al. (2005) cost reduction in cultural heritage recording was sought by utilising consumer-grade digital cameras. Although this achieves some savings, exterior orientation estimation relies on reference points and, therefore, a surveyor and professional equipment is necessary. Boochs et al. (2007) enhanced the usability of photogrammetry for non-experts by providing a device for easy stereo image acquisition 
with consumer-grade cameras. In their method, control is provided by coordinated reference points but measured object distances can also be used, when the restriction to scaled measurements is acceptable.

The cost of surveying has been avoided completely by approaches that are limited to scaled measurements. Although reference to a 3D national reference system is preferable, scaled measurements are considered sufficient for simple sites. The use of scale bars as a scale reference has been demonstrated in Chandler and Fryer (2005) and Bryan and Chandler (2008). In the latter a technique for rock-art recording by non-specialists was developed and tested. Non-specialist volunteers were successfully trained in applying this technique, which resulted in a further reduction in recording costs.

Fiani and Pistillo (2004) developed a portable system for recording sea cliffs where images were taken from a motorboat. It consists of a dual-frequency GNSS receiver, digital compass, and a digital consumer-grade camera. The compass includes inclinometers and is capable of measuring 3D orientation with a nominal accuracy of $0.5^{\circ}$ for heading and $0 \cdot 15^{\circ}$ to $2 \cdot 0^{\circ}$ for both pitch and roll. All components were fixed on an aluminium bar and the offsets between the components were calibrated. The system was first tested on a building where surveyed reference points were used to derive the exterior orientation in a conventional manner. A comparison of the results with values directly provided by GNSS and compass show large differences. The authors suggest that the result could be improved by enhancing the calibration procedure and including geometrical constraints.

Coppa et al. (2007) built a pedestrian Mobile Mapping System (MMS) using the same compass used in the recording system described previously, an equivalent GNSS receiver and a digital camera. The offsets of the system components were calibrated using a calibration field established on the outside wall of a building. When testing the system, an absolute path displacement of $0.6-1.5 \mathrm{~m}$ was achieved. Relative distance measurements yielded differences between $0 \cdot 2-0 \cdot 4 \mathrm{~m}$. These large differences were attributed to poor image geometry, poor image point measurement, poor lighting conditions, poor performance of GNSS in built-up areas, and deviations in the compass reading due to possible magnetic distortions.

\section{RECORDING SYSTEM}

The recording system presented here comprises a consumer-grade digital camera, a 3D orientation sensor, a survey-grade GNSS receiver, and a laptop for data recording (Fig. 1(a)). The camera, orientation sensor, and GNSS antenna are assembled in a purpose-built, rigid mounting frame made of aluminium (Fig. 1(b)). This frame constrains the components in position and orientation, to enable a stable and reliable offset calibration. For data collection the mounting frame was attached to a camera tripod. The camera, GNSS receiver and orientation sensor are now described in more detail. 
KIRCHHÖFER et al. Direct exterior orientation determination for a low cost heritage recording system

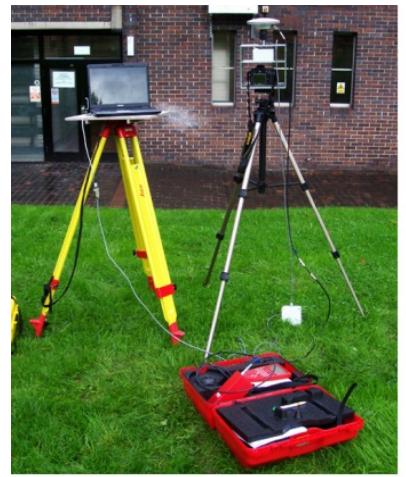

a: complete recording system

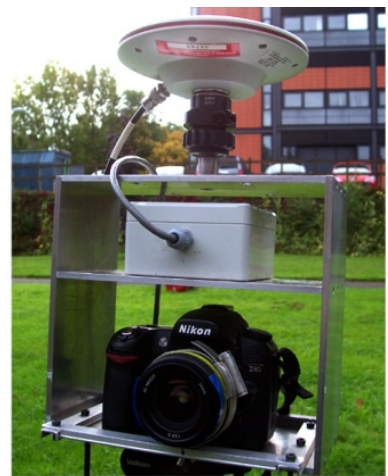

b: mounting frame

FIG. 1. Complete recording system and mounting frame.

\section{Camera}

For image acquisition a 10 megapixel Nikon D80 consumer-grade digital SLR camera was used. A Nikkor lens with a fixed focal length of $24 \mathrm{~mm}$ was attached. The camera was calibrated using a calibration field at Loughborough University. In order to maintain a degree of stability, the focus setting was fixed at infinity by wrapping electrical tape around the lens. The camera was attached to the mounting frame by a screw through the bottom of the mounting frame into the tripod socket of the camera. A second fixture involved a jubilee hose clip tightly clamped around the lens and fixed to the mounting frame, thus constraining the camera in its orientation.

\section{GNSS}

When the recording system was assembled in early 2010, no low-cost, small-size GNSS receivers were available on the market to provide the centimetre accuracy required in this project. Therefore, it was decided to use a survey-grade Leica System 500 dualfrequency GNSS receiver, capable of carrier phase measurements and enabling positioning with centimetre accuracy. Although this is certainly not a low-cost component (approximately $£ 8,000$ in 2006), it facilitates the testing of the principles of direct exterior orientation determination for close range photogrammetry. These principles will also be applicable when small-size, low-cost GNSS receivers for high accuracy positioning emerge on the market. The potential for this kind of GNSS receivers was subsequently demonstrated by the announcement of the SXBlue III (GENEQ Inc., 2010).

The GNSS antenna is rigidly fixed to the mounting frame while the GNSS receiver is connected to the antenna via a conventional cable. A prism can be fixed to the mounting frame and the position determined using a total station. This option facilitates the utilisation of the recording system when a GNSS receiver is not available or appropriate, for example inside buildings or under the canopy of forested areas. Testing of this option was already reported in Kirchhöfer et al. (2010) and is therefore not included in this paper. 
KIRCHHÖFER et al. Direct exterior orientation determination for a low cost heritage recording system

\section{Orientation Sensor}

The TCM5 (Fig. 2) of PNI Corporation is a MEMS-based 3D orientation sensor, utilising a three-axis magnetometer and a three-axis accelerometer that measures the heading, pitch, and roll angles. The accuracy specified by the manufacturer is $0 \cdot 3^{\circ}$ to $0 \cdot 5^{\circ}$ for heading, $0 \cdot 2^{\circ}$ for pitch, and $0 \cdot 2^{\circ}$ to $1 \cdot 0^{\circ}$ for roll, depending on the pitch angle (Table I). The cost of the sensor was $£ 1,600$ in 2008, enabling a low-cost 3D orientation determination.

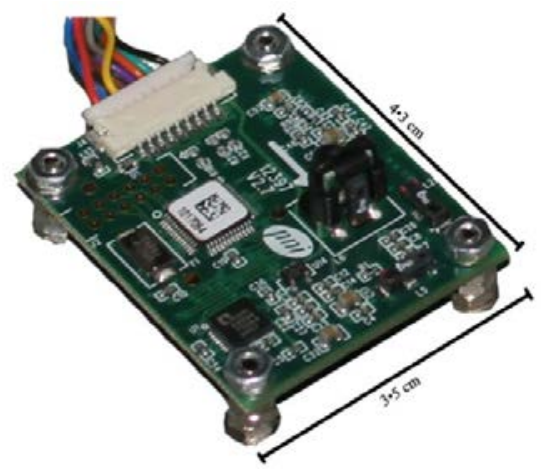

FIG. 2. TCM5 orientation sensor.

TABLE I. Accuracy specifications of TCM5.

\begin{tabular}{lcc}
\hline \multicolumn{1}{c}{ Angle } & Condition & Root mean square error $\left(^{\circ}\right)$ \\
\hline \multirow{2}{*}{ Heading } & Tilt $<70^{\circ}$ & $0 \cdot 3$ \\
\multirow{2}{*}{ Pitch } & Tilt $>70^{\circ}$ & $0 \cdot 5$ \\
& - & $0 \cdot 2$ \\
\multirow{2}{*}{ Roll } & Pitch $<65^{\circ}$ & $0 \cdot 2$ \\
& Pitch $<80^{\circ}$ & $0 \cdot 5$ \\
& Pitch $<86^{\circ}$ & $1 \cdot 0$ \\
\hline
\end{tabular}

The orientation sensor uses magnetometers for heading determination; thus measurements are influenced by local magnetic field distortions. These distortions can be caused by other components built in the recording system or by objects close to the recording system. To achieve high accuracy measurements it is necessary to measure these distortions using an initialisation process, during which the recording system is sequentially rotated and tilted in different orientations while the orientation sensor software measures the magnetic field strength. A built-in algorithm within the orientation sensor uses these measurements to estimate the distortions and to adjust subsequent measurements accordingly (PNI Corporation, 2009). The initialisation process has to be carried out prior to use.

The orientation sensor is connected to an off-the-shelf laptop that is used to operate the sensor and store the sensor measurements, utilising software provided by PNI Corporation (TCMStudio35). The output of the orientation sensor is a time coded list of continuous orientation measurements. To be able to retrieve the orientation measurement corresponding to each image, the internal clock of the camera has to be synchronised with 
the laptop clock, ideally before data collection. This is achieved using the synchronisation functionality of Nikon PictureProject software, provided with the Nikon D80 camera.

\section{TESTING}

For testing and calibrating the recording system, a test field was established on an outside wall of a building at Loughborough University's campus (Fig. 3). The field consists of 23 targets attached to the wall with an approximate separation of $3 \mathrm{~m}$. Two survey stations were established adjacent to the test field using differential GNSS positioning. Ordnance Survey National Grid (OSGB36) coordinates of the targeted points were determined using a total station and observations acquired from both survey stations. Three recording system performance tests were conducted on three different days.
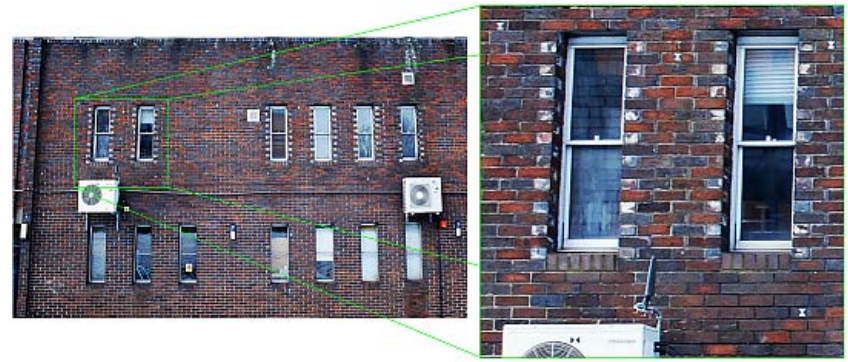

FIG. 3. Test field at Loughborough University’s campus

\section{Data Collection and Preparation}

During each test imagery, orientation and position data were recorded from a varying number of camera stations at the test site. A camera station is defined as the position of the camera's perspective centre and orientation of the camera at the time of image acquisition. Data was collected with camera-to-object distances varying from $7 \mathrm{~m}$ to $10 \mathrm{~m}$. The test field is approximately planar and so the range of heading values measured would be comparatively limited. To be able to test the recording system with a greater range of heading values that would be likely to occur in heritage recording projects, some images were deliberately taken with an oblique view in relation to the test field. For the same reason the range of pitch values was also deliberately increased during data collection. An overview of the number of images in each test, and the heading, pitch and roll ranges covered, can be found in Table II. Fig. 4 provides a simplified representation of the image configuration in the horizontal plane for Test1 and Test3. The image configuration in Test2 is comparable to Test3 and is omitted here due to space restrictions.

TABLE II. Number of images and range of heading, pitch and roll values of recording system tests.

\begin{tabular}{ccccc}
\hline Test & $\begin{array}{c}\text { Number of } \\
\text { images }\end{array}$ & $\begin{array}{c}\text { Heading range } \\
\left({ }^{\circ}\right)\end{array}$ & $\begin{array}{c}\text { Pitch range } \\
\left({ }^{\circ}\right)\end{array}$ & $\begin{array}{c}\text { Roll range } \\
\left({ }^{\circ}\right)\end{array}$ \\
\hline Test1 & 34 & $299 \cdot 4-351 \cdot 6$ & $-0 \cdot 4-22 \cdot 8$ & $-2 \cdot 9-6 \cdot 0$ \\
Test2 & 27 & $319 \cdot 0-338 \cdot 7$ & $1 \cdot 2-21 \cdot 2$ & $-1 \cdot 7-1 \cdot 3$
\end{tabular}



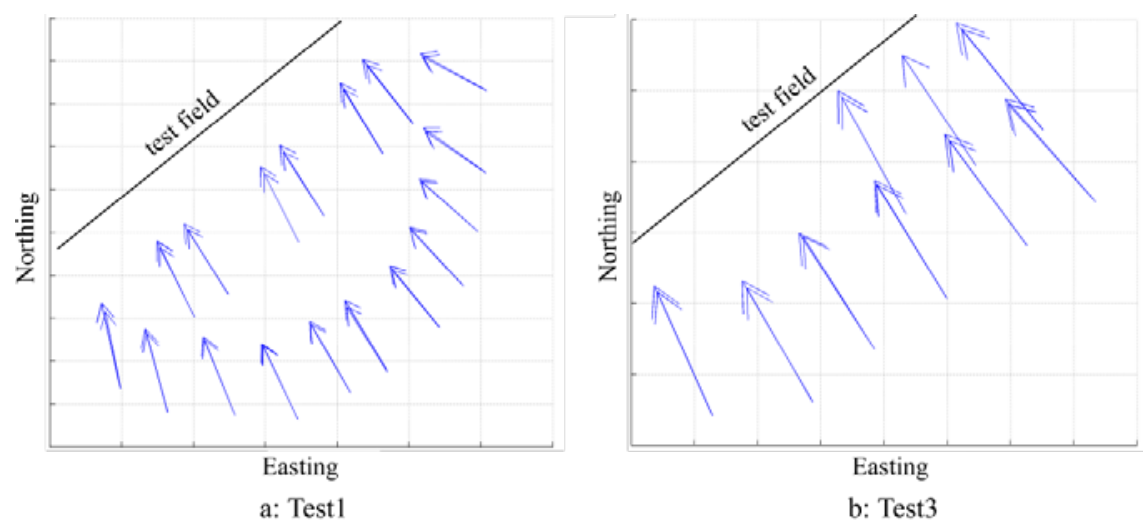

FIG. 4: Image configuration in the horizontal plane for Test1 and Test3.

Prior to data collection for each test, the internal clock of the camera was synchronised to the laptop clock and the orientation sensor was initialised at the location of the first camera station. During data collection the orientation sensor continuously recorded the heading, pitch and roll at intervals of 0.5 seconds, while the discrete GNSS positions were recorded for every camera station. A GNSS base station for differential post-processing had been set up at one of the survey stations adjacent to the test field.

The data collected at the test field was further processed as preparation for data analysis. This included post-processing of GNSS positions using Leica SKI-Pro software and transformation of the results into OSGB36 coordinates using Grid InQuest transformation software provided by Ordnance Survey (GB). Furthermore, heading, pitch and roll measurements were extracted from the continuous record of orientation sensor data using the exposure time information stored in the Exif header of each image. Local declination and convergence angles were applied to magnetic heading measurements in order to derive headings with respect to grid north. In a final step, the exterior orientation parameters for each image were derived indirectly in a bundle adjustment using Leica Photogrammetry Suite (LPS) version $9 \cdot 3$ where, typically, 10 to 20 coordinated points were measured in each image. These exterior orientation values are considered true and are used as a basis for calibrating the offsets between the components of the recording system.

\section{Offset Calibration}

To directly derive the exterior orientation of the camera, the rotational offsets between the camera and orientation sensor, and the positional offsets between the camera and GNSS antenna, need to be determined. Conventionally, calibration parameters are obtained in an expanded bundle adjustment (Cramer and Stallmann, 2002) using high-end and specialised software that is often expensive or inaccessible to non-specialists. However, this project seeks low-cost solutions for cultural heritage recording, which also includes approaches for offset calibration. Here, a simple method for offset calibration is 
presented that can easily be implemented by non-specialists using off-the-shelf software. This method has proven practicable for low-cost heritage recording, which will be demonstrated in the "Results" section.

Offset calibration was achieved by comparing the exterior orientation parameters $\left(\omega_{P}, \varphi_{P}, \kappa_{P}, X_{P}, Y_{P}, Z_{P}\right)$ derived indirectly in a bundle adjustment in LPS, with directly measured GNSS coordinates $\left(X_{D}, Y_{D}, Z_{D}\right)$ and orientation angles $\left(h_{D}, p_{D}, r_{D}\right)$. This estimates the three rotational and the three positional offset calibration values $(\Delta h, \Delta p, \Delta r, \Delta x, \Delta y, \Delta z)$ and also provides an indicator of the calibration precision. The calibration procedure was coded in Matrix Laboratory (MatLab) of MathWorks. The algorithm is described in more detail below.

One issue that complicated the rotational offset determination was the two different methods of describing rotation angles involved in the calculations. Heading $\left(h_{D}\right)$, pitch $\left(p_{D}\right)$ and roll $\left(r_{D}\right)$ are defined as independent rotation angles about the fixed axes of the orientation sensor coordinate system. The angle $h_{D}$ is the rotation angle around the z-axis and is always measured with respect to north. The angles $p_{D}$ and $r_{D}$ are the rotation angles around the $y$-axis and the $\mathrm{x}$-axis, respectively. Both are measured with respect to the local horizontal plane, which is defined by local gravity. In contrast, the angles normally used in photogrammetry, omega $\left(\omega_{P}\right)$, phi $\left(\varphi_{P}\right)$ and kappa $\left(\kappa_{P}\right)$, describe rotations about the non-fixed axes of the camera coordinate system. In this project the rotation sequence for the "normal case" of photogrammetry $(\omega, \varphi, \kappa)$ was used instead of the "terrestrial case" sequence $(\varphi, \omega, \kappa)$. The reason for this was that off-the-shelf photogrammetric software often does not support the terrestrial case of photogrammetry, which could prevent the implementation of the offset calibration approach presented in this paper. The alignment of the orientation sensor axes in the recording system's mounting frame with respect to the camera coordinate system is depicted in Fig. 5. Due to this alignment, changes in heading have a direct relationship with changes in the rotation angle value of $\varphi_{P}$ in the camera coordinate system. Changes in pitch and roll are strongly related to changes in $\omega_{P}$ and $\kappa_{P}$, respectively. 
KIRCHHÖFER et al. Direct exterior orientation determination for a low cost heritage recording system

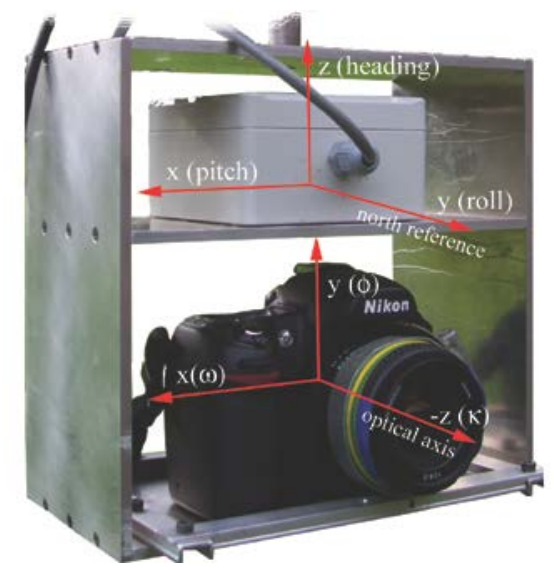

FIG. 5. Alignment of orientation sensor coordinate system with respect to the camera coordinate system (terrestrial orientation).

Using differing methods of describing rotation angles resulted in the need to find an approach to convert the angles of one system into equivalent angles in the other system. Conversion between photogrammetric and orientation sensor angles was achieved by creating a rotation matrix from angles of one system and extracting the angles of the other system from this matrix. For the angles $\omega, \varphi, \kappa$ the conventional rotation matrix for photogrammetry $\mathbf{R}_{\kappa \varphi \omega}$ was used:

$$
\mathbf{R}_{\kappa \varphi \omega}=\mathbf{R}_{\kappa} \mathbf{R}_{\varphi} \mathbf{R}_{\omega} .
$$

The orientation sensor angles describe rotations about the fixed axes of the orientation sensor system in the rotation sequence $(h, p, r)$. The rotation matrix $\mathbf{R}_{h p r}$ for these angles is derived as:

$$
\mathbf{R}_{h p r}=\mathbf{R}_{h} \mathbf{R}_{p} \mathbf{R}_{r} .
$$

The rotation matrix $\mathbf{R}_{h}$ describes a rotation about the z-axis by heading, $\mathbf{R}_{p}$ describes a rotation about the x-axis by pitch, and $\mathbf{R}_{r}$ describes a rotation about the y-axis by roll. Fig. 5 demonstrates that the camera is oriented in the terrestrial case of photogrammetry. Therefore, the rotational offset between terrestrial and normal case was included by utilising the rotation matrix $\mathbf{R}_{T N}$ that rotates the system from the terrestrial case into the normal case:

$$
\mathbf{R}_{T N}=\left[\begin{array}{ccc}
1 & 0 & 0 \\
0 & 0 & -1 \\
0 & 1 & 0
\end{array}\right] .
$$

For offset calibration, the photogrammetric exterior orientation angles of all camera stations $i\left(\omega_{P i}, \varphi_{P i}, \kappa_{P i}\right)$ were converted into their equivalent heading $\left(h_{P i}\right)$, pitch $\left(p_{P i}\right)$ and roll $\left(r_{P i}\right)$ values by inserting $\omega_{P i}, \varphi_{P i}, \kappa_{P i}$ into $\mathbf{R}_{\omega \varphi \kappa}$ and multiplying the inverse of this matrix by the inverse of $\mathbf{R}_{T N}$, resulting in the rotation matrix $\mathbf{R}_{t c i}$ : 
KIRCHHÖFER et al. Direct exterior orientation determination for a low cost heritage recording system

$$
\mathbf{R}_{t c i}=\mathbf{R}_{\kappa \varphi \omega}{ }^{-1} \mathbf{R}_{T N}{ }^{-1}=\left[\begin{array}{lll}
a_{11} & a_{12} & a_{13} \\
a_{21} & a_{22} & a_{23} \\
a_{31} & a_{32} & a_{33}
\end{array}\right] .
$$

The angles $h_{P i}, p_{P i}$, and $r_{P i}$ were extracted from $\mathbf{R}_{t c i}$ in equation (4) using equations (5) to (7):

$$
\begin{gathered}
h_{P i}=360-\arctan 2\left(\frac{-a_{12}}{a_{22}}\right) \\
p_{P i}=\arcsin \left(a_{32}\right) \\
r_{P i}=\arctan 2\left(\frac{-a_{31}}{a_{33}}\right)
\end{gathered}
$$

where $\arctan 2$ is the four-quadrant inverse tangent.

The pitch and roll offset calibration values $\Delta p$ and $\Delta r$ were calculated as the arithmetic means of all differences $\left(p_{P i}-p_{D i}\right)$ and $\left(r_{P i}-r_{D i}\right)$ respectively. The heading offset calibration value was derived in a slightly different way. Instead of averaging all differences, $\Delta h$ was calculated as the arithmetic mean of the differences $\left(h_{P i}-h_{D i}\right)$ for all $i$ that represent camera stations at the location of the orientation sensor initialisation. Between these stations the camera tripod was not moved and only the mounting frame was tilted to different degrees. This exception was due to recognition that magnetometer readings become increasingly invalid when the recording system is moved away from the location of orientation sensor initialisation. Quantification and correction of heading difference that were caused by moving the recording system would require measurement of the local magnetic field distortions at each location of data collection. This is not feasible for low-cost solutions that are practicable for non-specialists. However, the approach for deriving $\Delta h$ described above ensured that only heading measurements with a valid magnetometer gauging were used for offset calibration value determination.

The standard deviations of the heading $\left(\sigma_{h}\right)$, pitch $\left(\sigma_{p}\right)$, and roll $\left(\sigma_{r}\right)$ offset calibration values were also calculated. They indicate the calibration precision. In the case of $\Delta h$ the significance of the standard deviation $\sigma_{h}$ is reduced, due to the lower number of measurements used in the calculation of $\Delta h$. The heading calibration value was typically calculated using data from two camera stations, which was considered insufficient for yielding significant standard deviations. Therefore, it was decided to additionally derive the span or range of heading offsets $s \Delta h$, which is the maximum difference between $\Delta h$ and any value of $\left(h_{P i}-h_{D i}\right)$. This value indicates the suitability of $\Delta h$ as offset calibration value for heading measurements at camera stations that were not used to derive $\Delta h$.

The corrected orientation sensor angles $\left(h_{C i}, p_{C i}, r_{C i}\right)$ were calculated by adding $\Delta h$, $\Delta p$ and $\Delta r$ to all of their respective orientation sensor measurements $h_{D i}, p_{D i}$ and $r_{D i}$. The final step in the rotational offset calibration was the conversion of $h_{C i}, p_{C i}, r_{C i}$ into photogrammetric angles $\omega_{C i}, \varphi_{C i}, \kappa_{C i}$. This was achieved by inserting $\left(360^{\circ}-h_{C i}\right), p_{C i}$ and $r_{C i}$ in $\mathbf{R}_{h p r}$ in equation (2) and multiplying this matrix by $\mathbf{R}_{T N}$ in equation (3) in order to derive the rotation matrix $\mathbf{R}_{n c i}$ that relates to the normal case of photogrammetry: 
KIRCHHÖFER et al. Direct exterior orientation determination for a low cost heritage recording system

$$
\boldsymbol{R}_{n c i}=\boldsymbol{R}_{h p r} \boldsymbol{R}_{T N}=\left[\begin{array}{lll}
r_{11} & r_{12} & r_{13} \\
r_{21} & r_{22} & r_{23} \\
r_{31} & r_{32} & r_{33}
\end{array}\right] .
$$

From $\mathbf{R}_{n c i}$ in equation (8) the angles $\omega_{C i}, \varphi_{C i}, \kappa_{C i}$ were derived as follows:

$$
\begin{gathered}
\omega_{C i}=\arctan 2\left(\frac{-r_{23}}{r_{33}}\right) \\
\varphi_{C i}=\arcsin \left(r_{13}\right) \\
\kappa_{C i}=\arctan 2\left(\frac{-r_{12}}{r_{11}}\right) .
\end{gathered}
$$

For determination of the positional offset calibration values, it has to be considered that absolute positional offsets between the camera and the GNSS antenna change due to varying orientation angles during data collection. Therefore, the relative offsets for all camera stations $i$ were derived by rotating all absolute positional offsets $\mathbf{d}_{a i}=$ $\left[\left(X_{P i}-X_{D i}\right)\left(Y_{P i}-Y_{D i}\right)\left(Z_{P i}-Z_{D i}\right)\right]^{\mathrm{T}}$ into the non-rotated camera coordinate system, resulting in relative positional offsets $\mathbf{d}_{0 i}=\left[\begin{array}{lll}X_{0 i} & Y_{0 i} & Z_{0 i}\end{array}\right]^{\mathrm{T}}$. For this rotation, a rotation matrix $\mathbf{R}_{0 i}$ derived by inserting the exterior orientation angles $\omega_{P i}, \varphi_{P i}$ and $\kappa_{P i}$ into $\mathbf{R}_{\kappa \varphi \omega}$ in equation (1) was utilised. Fig. 6 depicts a simplified illustration of positional offsets in the rotated and non-rotated camera coordinate systems.

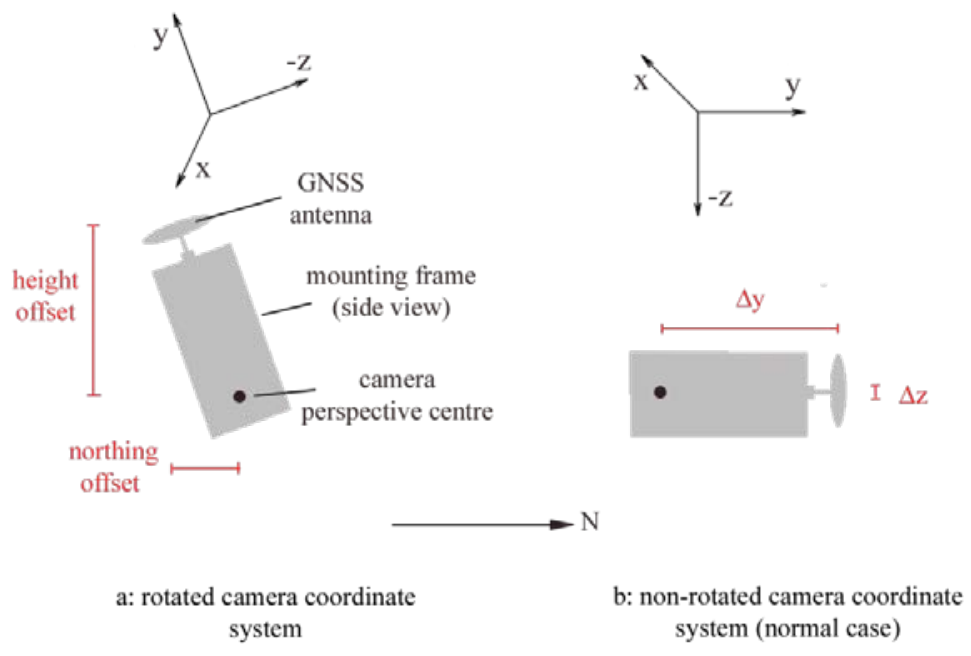

FIG. 6. Simplified representation of positional offset values in the rotated (left) and non-rotated (right) camera coordinate system. 
KIRCHHÖFER et al. Direct exterior orientation determination for a low cost heritage recording system

The calibration values for the positional offsets $(\Delta x, \Delta y, \Delta z)$ were calculated as the arithmetic means of $X_{0 i}, Y_{0 i}$ and $Z_{0 i}$, respectively. The standard deviations $\sigma_{x}, \sigma_{y}$ and $\sigma_{z}$ were calculated as an indicator of calibration precision. To correct the GNSS measurements for positional offsets, absolute calibration values $\mathbf{c}_{a i}=\left[\begin{array}{lll}\Delta x_{a i} & \Delta y_{a i} & \Delta z_{a i}\end{array}\right]^{\mathrm{T}}$ for each camera station $i$ were derived by rotating the vector of relative calibration values $\mathbf{c}_{0}=\left[\begin{array}{lll}\Delta x & \Delta y & \Delta z\end{array}\right]^{\mathrm{T}}$ into the rotated camera coordinate system. The rotation matrix $\mathbf{R}_{a i}$ used for this purpose was derived from the corrected and converted orientation sensor angles $\omega_{C i}, \varphi_{C i}, \kappa_{C i}$ using the inverse of $\mathbf{R}_{\kappa \varphi \omega}$. The corrected GNSS positions $\left(X_{C i}, Y_{C i}, Z_{C i}\right)$ are calculated by adding $\Delta x_{a i}, \Delta y_{a i}$ and $\Delta z_{a i}$ to their respective GNSS measurements $X_{D}, Y_{D}$ and $Z_{D}$.

\section{Accuracy Assessment}

The corrected orientation sensor and GNSS measurements were used to provide initial exterior orientation parameters in the bundle adjustment software GAP (Chandler and Clarke, 1992). Except for $\varphi$, the exterior orientation parameters were constrained by their respective standard deviation from the offset calibration process, allowing them to vary within these limits. The value of $\varphi$ is strongly related to the measured heading value (Fig. 5). The heading calibration value $\Delta h$ was derived solely from data of camera stations at the location of orientation sensor initialisation. As a result $\Delta h$ was not considered ideal for correcting heading measurements at other camera stations, due to potential erroneous heading measurements caused by local magnetic field distortions. Therefore, $\varphi$ is constrained by two different values, depending on the location where the corresponding heading data was collected. For the camera stations at the initialisation location, the expected sensor heading accuracy (Table I) was used. For all other camera stations the range of heading offsets $s \Delta h$ was used. This reduces the effect of the camera stations where the heading offset calibration is less reliable on the bundle adjustment process. In the bundle adjustment no control points were used, relying on the direct exterior orientation parameters only. The targeted points of the test field were used as check points and their coordinates were estimated in the bundle adjustment. These coordinates were compared to the original coordinates of the points, thus allowing the calculation of the root mean square error (RMSE) to quantify absolute accuracy. Relative accuracy was also assessed. 3D distances between all possible pairs of targeted points were calculated from the estimated check point coordinates. These distances were compared to distances calculated from the original target point coordinates. The RMSE of the distance differences indicates the relative accuracy.

The aim of this project is to develop a system that meets the requirements for medium accuracy heritage recording. These requirements depend upon the type of heritage object and the defined end product. However, the boundaries between high, medium, and low accuracy are difficult to define (Wackrow, 2008). English Heritage's 'Metric survey specifications for cultural heritage' (English Heritage, 2009) define accuracy requirements ranging from $9 \mathrm{~mm}$ to $15 \mathrm{~mm}$ for recording buildings and immovable objects. This can certainly be considered high accuracy when recording comparatively large, immovable objects. In relation to this, medium accuracy in this project is defined as achieving measurements that deviate from their true values by 25 $\mathrm{mm}$ to $40 \mathrm{~mm}$. 
KIRCHHÖFER et al. Direct exterior orientation determination for a low cost heritage recording system

\section{RESULTS}

\section{Calibration Precision}

The standard deviations of the calibration values provide useful indicators for calibration precision. Standard deviations larger than the expected accuracy of orientation and position measurements indicate significant offset variations during data collection. Table III presents the standard deviation for rotational and positional offset calibration, and the range of heading offsets $s \Delta h$ for Test1 to Test3.

The standard deviation for heading $\sigma_{h}$ is comparatively small, with values more than $50 \%$ smaller than the specified heading accuracy (Table I). These small values were expected, because $\sigma_{h}$ is derived from data of typically two camera stations, which provides only a small sample for standard deviation calculation. The standard deviations for pitch and roll are acceptable, being smaller than the accuracy specifications. The range of heading offset indicates the suitability of the heading offset calibration value to correct all heading measurements of a dataset. All test datasets have a comparatively small maximum heading difference with the smallest value meeting the orientation sensor heading accuracy of $0.3^{\circ}$ and the largest value exceeding the accuracy by $0 \cdot 1^{\circ}$. This indicates only small changes in the heading offset due to changes in the local magnetic field.

TABLE III. Standard deviations of rotational and positional offset calibration and range of heading offset.

\begin{tabular}{cccccccc}
\hline & $\sigma_{h}\left(^{\circ}\right)$ & $\sigma_{p}\left({ }^{\circ}\right)$ & $\sigma_{r}\left(^{\circ}\right)$ & $s \Delta h\left(^{\circ}\right)$ & $\sigma_{x}(\mathrm{~mm})$ & $\sigma_{y}(\mathrm{~mm})$ & $\sigma_{z}(\mathrm{~mm})$ \\
\hline Test1 & $0 \cdot 08$ & $0 \cdot 10$ & $0 \cdot 19$ & $0 \cdot 40$ & 14 & 26 & 17 \\
Test2 & $0 \cdot 05$ & $0 \cdot 10$ & $0 \cdot 20$ & $0 \cdot 35$ & 11 & 15 & 15 \\
Test3 & $0 \cdot 15$ & $0 \cdot 07$ & $0 \cdot 14$ & $0 \cdot 27$ & 7 & 20 & 13 \\
\hline
\end{tabular}

For data collection the recording system was oriented approximately vertically. Fig. 6 demonstrates that with this orientation the absolute height offset derived in the rotated camera system is the main contributor to the y-offset in the non-rotated camera system, which was used to derive the calibration value and standard deviation. Therefore, $\sigma_{y}$ was assessed using the expected GNSS height accuracy as reference. The expected positional accuracy of GNSS using carrier phase measurements is $10 \mathrm{~mm}$ in plan and $30 \mathrm{~mm}$ in height (Konecny, 2003). The two largest standard deviations for the positional offset calibration occur for $\sigma_{y}$, but they meet the expected GNSS height accuracy. The standard deviations $\sigma_{x}$ and $\sigma_{z}$ are assessed by comparing them to the expected GNSS accuracy in plan. In most cases the planimetric accuracy level is exceeded, indicating significant offset variations during data collection.

\section{Calibration Stability}

Calibration stability demonstrates the validity of calibration values over the two weeks in which the three tests were conducted. Stable offset calibration is achieved when changes between calibration values do not exceed the expected measurement accuracy. The rotational offset calibration values of Test 1 to Test 3 are shown in Fig. 7(a). The small increase of the absolute heading value from Test $1\left(-5 \cdot 11^{\circ}\right)$ to Test3 $\left(-5 \cdot 78^{\circ}\right)$ marks the largest calibration value change between the test datasets. The magnitude of the change 
$\left(0 \cdot 67^{\circ}\right)$ exceeds the expected heading accuracy of $0 \cdot 3^{\circ}$ (Table I). The calibration values for pitch and roll appear more stable. The largest change for pitch is just $0 \cdot 15^{\circ}$ and for roll is $0 \cdot 04^{\circ}$, which are both smaller than the orientation sensor accuracy specification.

With regard to the positional offset calibration values, $\Delta y$ varies most, changing from $-248 \mathrm{~mm}$ to $-219 \mathrm{~mm}$ between Test1 and Test2 (Fig. 7(b)). The magnitude of the offset change (28 mm) does meet the expected accuracy for GNSS height measurements. The largest change for $\Delta x$ occurs between Test2 $(-27 \mathrm{~mm})$ and Test3 $(-8 \mathrm{~mm})$. This magnitude (19 mm) exceeds the expected planimetric accuracy of GNSS. $\Delta z$ is the most stable calibration value with the largest change being $3 \mathrm{~mm}$.
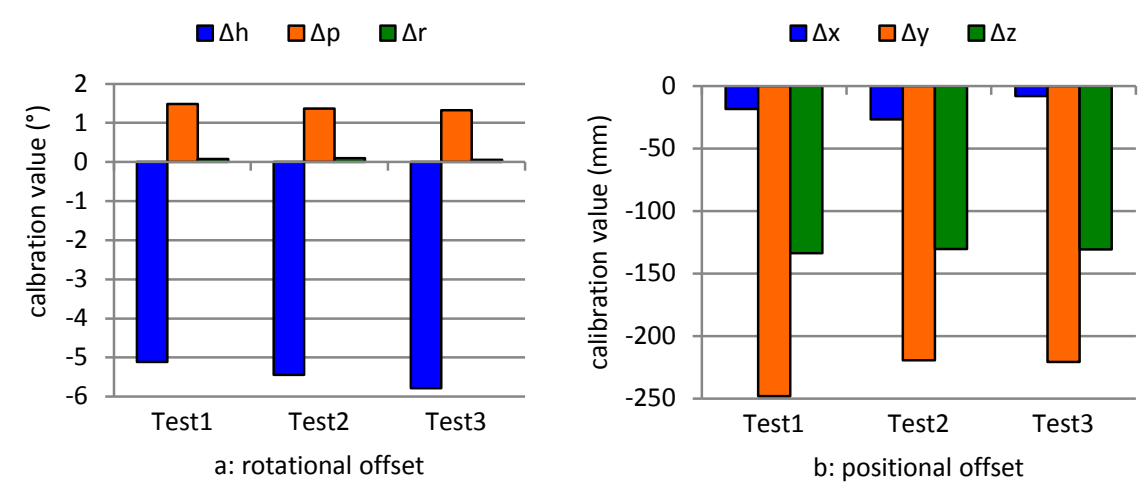

FIG. 7. Rotational (a) and positional (b) offset calibration values.

\section{Absolute Accuracy}

Offset calibration for each of the three test data sets resulted in three different sets of calibration values. Each of these calibration value sets was applied to measurements of the two other datasets. This resulted in six sets of directly determined exterior orientation parameters that were used in a bundle adjustment using the GAP software. The RMSE of the differences between object coordinates of check points estimated in the bundle adjustment and their true coordinates is an indicator of the absolute accuracy. The RMSE for the different test datasets and calibration values combinations is depicted in Fig. 8. The RMSE is worst when the calibration values of Test1 are used to correct the measurements in Test3, with the largest value being $40 \mathrm{~mm}$. In all other combinations the RMSE is smaller than $21 \mathrm{~mm}$, with most values not exceeding $16 \mathrm{~mm}$. The result with the best RSME is achieved with the calibration value derived from Test2 applied to the measurements in Test3. These results demonstrate that the recording system can provide data with an absolute positional accuracy of $40 \mathrm{~mm}$ in the object space.

\section{Relative Accuracy}

The relative or inner accuracy quantifies the recording system's capability to provide data for measurements that are accurate in relation to each other. This was assessed by comparing 3D distances between check point coordinates estimated in a GAP bundle adjustment with equivalent distances derived from the original coordinates. The RMSE of the distance differences indicates the relative accuracy and is depicted in Fig. 9. The 3D 
relative accuracy achieved for different combinations of calibration values and test datasets ranges from $3 \mathrm{~mm}$ to $10 \mathrm{~mm}$ with most values smaller than $8 \mathrm{~mm}$. These values are significantly better than the values of absolute accuracy. As perhaps expected, this means that object points can be more accurately defined relative to each other than their absolute position in a national reference system.

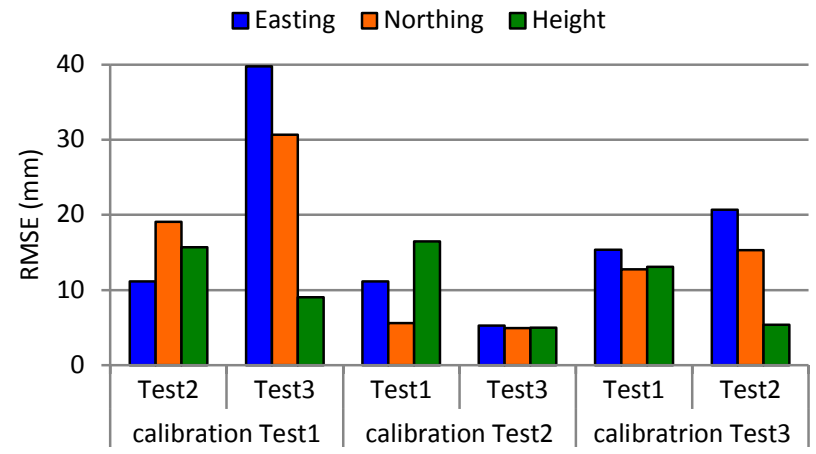

FIG. 8. Absolute accuracy (RMSE) achieved for different test datasets and calibration combinations.

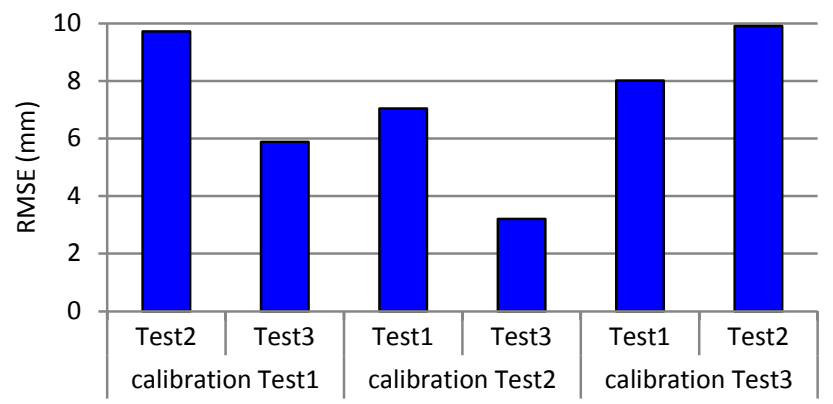

FIG. 9. Relative accuracy (RMSE) achieved for different test datasets and calibration combinations..

\section{DISCUSSION}

The calibration results conveyed by Fig. 7(a) imply some instability of the component fixture within the mounting frame, despite the measures taken to fix the camera in the frame. The change in heading offset is significantly larger than the accuracy defined in the orientation sensor specifications (Table I). At the same time the range of heading offsets $s \Delta h$ is comparatively low (Table III), indicating that the changes occur between data collections. Considering that the orientation sensor is contained in a box that is fixed with four screws to the mounting frame, the camera fixture is probably the most sensitive to physical strain due to handling or transportation of the recording system. Some components of the camera fixture, for example the jubilee hose clip, are made of comparatively thin material and could deform when the camera fixture is physically 
strained. This demonstrates the difficulty of fixing the orientation of a consumer-grade camera with respect to the recording system mounting frame. However, rotational calibration stability can certainly be improved by using more rigid camera fixture components that are less likely to deform.

Fixing the position of a consumer-grade camera is unproblematic, because utilising the camera tripod socket prevents discernible shifts of the camera. Therefore, the change in $\Delta x$ (Fig. 7(b)), which exceeds the expected planimetric GNSS accuracy, cannot be attributed to instability of the camera fixture. The magnitude of this change could be explained by a slight decrease in GNSS accuracy due to tilting the antenna during data collection. This is also indicated by the standard deviation $\sigma_{x}$ exceeding the theoretical planimetric GNSS accuracy (Table III). According to Kirk (2010), tilting the antenna can result in a GNSS ranging error of up to $10 \mathrm{~mm}$ due to unsuitable antenna design. Tilting the antenna also increases the effect of multipath error, which can further degrade GNSS positioning. This issue needs to be solved for similar recording systems in the future, in order to allow recording of heritage objects that require larger tilts of the mounting frame, for example objects with low elevation angles from the ground, such as horizontal rockart panels.

Despite the changes in rotational and positional offset calibration values, direct determination of exterior orientation parameters can achieve absolute accuracies of $40 \mathrm{~mm}$. This value was obtained when applying calibration values of Test1 to direct measurements of Test3. In other combinations an RMSE of $21 \mathrm{~mm}$ and smaller was achieved. Further investigation revealed that the decreased accuracy when applying calibration values of Test1 to measurements of Test 3 was caused by the significant heading calibration value change of $0.67^{\circ}$ between Test 1 and Test 3 . However, when calibration values of Test 3 were applied to direct measurements of Test 1 an RMSE of $15 \mathrm{~mm}$ was achieved. This indicates that the errors in the direct exterior orientation parameters due to heading calibration value change could be compensated during bundle adjustment in Test1 but not in Test3. This can be explained by the image configuration in the horizontal plane, which is more convergent in Test1 than in Test3 (Fig. 4). This indicates that erroneous heading corrections due to unstable offset calibration can be compensated during the bundle adjustment when data is collected with a convergent image configuration.

In order to enhance comprehension, some further investigations into achievable absolute accuracy were conducted. The RMSE of coordinate differences represents positional offsets between estimated and true coordinates. These offsets can be considered as a result of a 3D shift, a 3D rotation, and a scale difference between a block of estimated coordinates and a block of true coordinates. Using a 3D similarity transformation, three shifts, three rotations and one scale factor were derived for the six combinations of calibration values and direct measurements. The results revealed that the three shift factors in some cases exceeded the expected GNSS measurement accuracy (10 $\mathrm{mm}$ in plan and $30 \mathrm{~mm}$ in height), but are of similar magnitude as the standard deviations of the positional offset calibration (Table III). This reflects the decrease in GNSS accuracy due to antenna tilt. In most cases the three rotation factors did not exceed the expected orientation sensor measurement accuracy. In a few instances the expected accuracy was exceeded, which is certainly caused by offset calibration value changes. This demonstrates that the achievable absolute accuracy is within the expected limits set by the orientation sensor and GNSS measurement accuracy, when stable offset calibration 
is achieved. Therefore, orientation sensor and GNSS accuracy can be considered the main contributors to the achievable overall system accuracy. The scale factors obtained for the 6 blocks of estimated check point coordinates were close to unity $(0.9984-1.0009)$.

For cultural heritage recording the relative accuracy is of high significance also, because it provides information about how accurately object points can be related to each other. The relative accuracy achieved in the recording system tests is significantly better than the absolute accuracy implies. This demonstrates that recorded data can be related to a national reference frame with acceptable accuracy, whilst spatial relationships between features are maintained to a considerably better accuracy.

The findings are in contrast of those of Fiani and Pistillo (2004) who achieved poor results with their recording system. The results of accuracy assessment achieved in our study demonstrate the capability of the recording system to directly determine the exterior orientation of a camera. Absolute and relative accuracy both indicate the usability of the recording system for many cultural heritage recording projects. Results of a case study conducted by the lead author at St. Catherine's Oratory on the Isle of Wight, UK, verified this (Kirchhöfer et al., 2011).

With the focus on low-cost and easy-to-use components, the recording system could satisfy demands for a simple and cost-effective method for heritage recording. It also has potential to enhance the recording methods developed and tested by Chandler and Fryer (2005) and Bryan and Chandler (2008) by providing information to relate extracted data to a national reference system without the need for coordinated control points. The recording system and offset calibration method developed in this project are expected to be usable by non-specialists. The ability of non-specialists to carry out image acquisition and photogrammetric processing using simple and low-cost approaches was already noted in Bryan and Chandler (2008). Nevertheless, the practicability of the recording system could be further enhanced by some alterations to the system. Stabilising the camera fixture to the mounting frame avoids offset changes due to handling or transportation and results in an increase of achievable absolute accuracy. Another alteration is to replace the survey-grade GNSS receiver with a low-cost and small-size device that achieves positioning with centimetre accuracy. This will help to achieve the ultimate goal of developing a proper low-cost recording system which is portable, lightweight, and easyto-use.

A key question, however, is whether the approach described in this paper remains the right approach. The recent and rapid development of Structure from Motion (SfM) and MultiView Stereo (MVS) approaches now embedded in products like PhotoSynth and 123D Catch, provide easy-to-use and free solutions which could be used for recording heritage. These were not available at the time this doctoral programme was conceived in mid-2008 but do not generate data oriented in a national coordinate reference system. If this is an important requirement then the approach developed here does have a critical role and provides the theoretical framework for recording heritage using SmartPhone technology with embedded MEMS orientation and GNSS position data. Recent tests conducted at Loughborough University suggest that the "missing link" is more accurate positional information, which must surely come (Kirchhöfer et al., 2011). 
KIRCHHÖFER et al. Direct exterior orientation determination for a low cost heritage recording system

\section{CONCLUSION}

The results presented in this paper demonstrate that in an image-based recording system combined with direct exterior orientation determination, it is possible to achieve absolute accuracy levels in object space of $40 \mathrm{~mm}$ and relative accuracy levels of $10 \mathrm{~mm}$ and better. The recording system is therefore believed to be suitable for many cultural heritage recording tasks requiring data directly referenced to a national coordinate reference system. However, to achieve a proper low-cost recording system, an inexpensive device for positioning with centimetre accuracy has to replace the surveygrade GNSS receiver. The changes in the offset calibration values also showed that enhancements in the recording system component fixtures to the mounting frame are necessary to enable a more stable offset calibration. This certainly holds the greatest potential for improving the recording system, especially when the issue of decreasing GNSS accuracy due to tilting the system can be solved. It is expected that a more stable offset calibration will result in an achievable absolute accuracy of $20 \mathrm{~mm}$.

\section{ACKNOWLEDGEMENTS}

The authors wish to acknowledge the investment in a TCM5 orientation sensor by English Heritage, which made this project possible.

\section{REFERENCES}

Arias, P., Herráez, J., LOREnZo, H. and OrdóñEZ, C., 2005. Control of structural problems in cultural heritage monuments using close-range photogrammetry and computer methods. Computers and Structures, 83(21-22): 1754-1766.

Boochs, F., HeinZ, G., Huxhagen, U. and MüLler, H., 2007. Low-cost image based system for non-technical experts in cultural heritage documentation and analysis. CIPA 2007 XXI International Symposium, Athens, Greece. 6 pages (on CD-ROM).

BosCH, R., KÜLÜR, S. and GÜLCH, E., 2005. Non-metric camera calibration and documentation of historical buildings. CIPA 2005 XX International Symposium, Turin, Italy. 6 pages (on CD-ROM).

BRYAN, P. and CHANDLER, J. H., 2008. Cost-effective rock-art recording within a non-specialist environment. International Archives of Photogrammetry, Remote Sensing and Spatial Information Sciences, 37(B5): 259264.

ChAndleR, J. H. and ClARK, J. S., 1992. The archival photogrammetric technique. Further applications and development. Photogrammetric Record, 14(80): 241-247.

ChANDleR, J. H. and FRYER, J. G., 2005. Recording Aboriginal rock art using cheap digital cameras and digital photogrammetry. CIPA 2005 XX international Symposium, Turin, Italy. 6 pages (on CD-ROM).

CIPA, 2010. http://cipa.icomos.org/OBJECTIVES.HTML [Accessed 4th April 2011].

Clowes, M, 2002. Digital photogrammetry at English Heritage: a pictorial review of projects to date. Photogrammetric Record, 17(99): 441-452.

Coppa, U., Guarnieri, A., Pirotti, F. And Vettore, A., 2007. A backpack MMS application. 5th International Symposium on Mobile Mapping Technology, Padua, Italy, 7 pages (on CD-ROM).

CRAMER, M. AND STALLMANN, D., 2002. System calibration for direct georeferencing. International Archives of Photogrammetry, Remote Sensing and Spatial Information Sciences, 34(Part 3 A+B): A79-A84.

ENGLISH HERITAGE, 2009. Metric survey specifications for cultural heritage. Second edition, English Heritage, Swindon, UK. 126 pages.

FIANi, M. and PISTILLO, P., 2004. A low-cost MMS integrating GPS, digital compass and a camera to the direct georeferencing of digital images. International Archives of Photogrammetry, Remote Sensing and Spatial Information Sciences, 35(5): 747-752.

GENEQ INC., 2010. http://www.sxbluegps.com/SXBlue-III-version1.1.pdf [Accessed 12th January 2011].

Guarnieri, A., Menin, A., Pirotti, F. and Vettore, A., 2008. Low cost system: GPS/MEMS for positioning. Proceedings of FIG Working Week 2008, Stockholm, Sweden: 3020-3030. 
KirchhÖFER, M. K., ChandleR, J. H. and WACKrow, R., 2010. Testing and application of a low-cost photogrammetric recording system suitable for cultural heritage recording. RSPSoc Annual Conference 2010, Cork, Ireland. 8 pages (on CD-ROM).

KIRCHHÖFER, M. K., CHANDLER, J. H. and WACKROW, R., 2011. Cultural heritage recording utilising low-cost close-range photogrammetry. CIPA XXIII International Symposium 2011, Prague, Czech Republic. 8 pages (on CD-ROM).

KIRK, G. R., 2010. Tilt compensation for GNSS antenna. United States Patent 20100283674, published application. 16 pages.

KONECNY, G., 2003. Geoinformation. remote sensing, photogrammetry and geographic information systems. First edition. Taylor \& Francis, London, UK. 248 pages.

NiU, X., Hassan, T., Ellum, C. and El-Sheimy, N., 2006. Directly georeferencing terrestrial imagery using MEMS-based INS/GNSS integrated systems. XXIII FIG Congress, Munich, Germany. 16 pages (on CDROM).

Palumbo, G. and OGleby, C. L., 2004. Heritage at risk and CIPA today: a report on the status of heritage documentation. International Archives of Photogrammetry, Remote Sensing, and Spatial Information Sciences, 33(5): 239-842.

PNI CORPORATION, 2009. User Guide TCM3 \& TCM5. PNI Corporation, Santa Rosa, CA, USA. 58 pages.

Power of Place Office and English Heritage, 2000. Power of Place: The future of the historic environment. Power of Place Office, London, UK. 50 pages.

SCHWIEGER, V. and GLÄSER, A., 2005. Possibilities of low cost GPS technology for precise geodetic applications. Proceedings of FIG Working Week 2005, Cairo, Egypt. 16 pages (on CD-ROM).

UNESCO, 1972. http://whc.unesco.org/archive/convention-en.pdf [Accessed 5th May 2009].

WACKROW, R., 2008. Spatial measurement with consumer grade digital cameras. Ph.D. thesis, Loughborough University, UK. 196 pages.

WACKRow, R, CHANDlER, J. H. and BryAn, P., 2007. Geometric consistency and stability of consumer-grade digital cameras for accurate spatial measurement. Photogrammetric Record, 22(118): 121-134.

Wing, M. G., EKLund, A. and KellogG, L. D., 2005. Consumer-grade global positioning system (GPS) accuracy and reliability. Journal of Forestry, 103(4): 169-173.

YilmaZ, H. M., YAKAR, M., GulEc, S. A. and Dulgerler, O. N., 2007. Importance of digital close-range photogrammetry in documentation of cultural heritage. Journal of Cultural Heritage, 8(4): 428-433.

\section{Résumé}

\section{Zusammenfassung}

Nahbereichsphotogrammetrie hat sich als kostengünstige und leicht zu bedienende Technik für die Erfassung von Kulturdenkmälern bewährt. Handelsübliche Digitalkameras können genutzt werden, um schnell und kostengünstig Daten zu erfassen und es Laien $\mathrm{zu}$ ermöglichen, sich verstärkt für ihre lokalen Kulturdenkmäler zu engagieren, welches ein wesentliches Bestreben dieser Zusammenarbeit von English Heritage und der Loughborough University ist. Um dies zu verwirklichen und gleichzeitig Messungen in einem nationalen 3D-Bezugssystem zu gewährleisten, wurde ein Datenerfassungssystem entwickelt, welches geeignet ist, die äußere Orientierung während der Bildaufnahme direkt und kostengünstig herzuleiten. Das System besteht aus einer Digitalkamera, einem kleinen 3D-Orientierungssensor und einer GNSSAntenne. Diese sind in einem Befestigungsrahmen montiert, der die Kalibrierung des Versatzes zwischen den Komponenten ermöglicht. Testfelddaten wurden genutzt, um Präzision und Stabilität der Kalibrierung sowie erzielbare absolute und relative Genauigkeit zu untersuchen. Die Ergebnisse der Datenauswertung zeigen, dass das System, sogar mit leicht instabilen Versatzkalibrierungswerten, die 
KIRCHHÖFER et al. Direct exterior orientation determination for a low cost heritage recording system

Genauigkeitsanforderungen für die Denkmalerfassung mit mittlerer Genauigkeit erfüllen kann.

Resumen 